\title{
Comparison of two regimens for patients with thyroid-associated ophthalmopathy receiving intravenous methyl prednisolone: A single center prospective randomized trial
}

\author{
PAN-WEI MU ${ }^{1 *}$, XI-XIANG TANG ${ }^{2 *}$, YI-NA WANG $^{2}$, SHUO LIN $^{1}$, MAN-MAN WANG $^{1}$, QIONG-LI YIN ${ }^{2}$, \\ JIONG SHU ${ }^{1}$, BI-LIAN ZHU ${ }^{2}$, JING-REN LI ${ }^{1}$, LI ZHOU ${ }^{1}$, LONG-YI ZENG $^{1}$ and YAN-MING CHEN ${ }^{1}$ \\ ${ }^{1}$ Department of Endocrinology; ${ }^{2}$ Advanced Medical Center, The Third Affiliated Hospital of \\ Sun Yat-sen University, Guangzhou, Guangdong 510630, P.R. China
}

Received October 4, 2019; Accepted April 1, 2020

DOI: $10.3892 /$ etm.2020.9282

\begin{abstract}
Intravenous (i.v.) glucocorticoid is recommended for active moderate-to-severe thyroid-associated ophthalmopathy (TAO). However, the details of the treatment schedule are still debatable. The present prospective randomized trial was performed to compare clinical outcomes and serum cytokines between the two regimens. A cohort of 90 patients with active moderate-to-severe TAO was randomized to receive i.v. methyl prednisolone on a weekly protocol or daily scheme. The response rate was evaluated at the 12-week follow-up visit. Serum interleukin (IL)-2, IL-6 and IL-17 levels were measured in 160 patients with TAO, 60 patients with isolated Graves' disease (GD) and 60 normal control (NC) at baseline, as well as patients with active moderate-to-severe TAO at the 12th week after treatment. The daily scheme had a higher response rate than the weekly protocol without a significant difference (77.8 vs. $63.6 \%, \mathrm{P}>0.05)$. No major adverse events were recorded under either regimen. Overall, minor events were more common on the daily scheme $(11.36$ vs. $4.35 \%, \mathrm{P}<0.05)$ than on the weekly protocol, whereas the deterioration of eye symptoms (two patients) was only reported on the weekly protocol. At baseline, the IL-17 level in the TAO group was higher than that in the isolated GD and NC groups $(\mathrm{P}<0.05)$. In addition,
\end{abstract}

Correspondence to: Professor Yan-Ming Chen, Department of Endocrinology, The Third Affiliated Hospital of Sun Yat-sen University, 600 Tianhe Road, Guangzhou, Guangdong 510630, P.R. China

E-mail: chyanm@mail.sysu.edu.cn

${ }^{*}$ Contributed equally

Abbreviations: i.v., intravenous; TAO, thyroid-associated ophthalmopathy; GD, Graves' disease; NC, normal control; EUGOGO, European Group on Graves' Ophthalmopathy; CAS, clinical activity score

Key words: thyroid-associated ophthalmopathy, clinical activity score, glucocorticoid therapy, interleukin-2, interleukin-6, interleukin-17 the IL-17 level in the active TAO group was higher than that in the inactive TAO group $(\mathrm{P}<0.05)$. Furthermore, the IL-17 level had significantly decreased under the two regimens at the 12 -week visit $(\mathrm{P}<0.05)$. In conclusion, for patients with active moderate-to-severe TAO, daily i.v. glucocorticoid therapy has a relative higher response rate than the weekly protocol with a few more minor adverse events. These two regimens have their own merits with regard to adverse effects. IL-17 has the potential to be a biomarker for evaluating TAO activity and treatment effects.

\section{Introduction}

Thyroid-associated ophthalmopathy (TAO) is one of the most common extra-thyroidal manifestations of Graves' disease (GD). It is frequently mild and self-limiting and only requires observation or local treatment. However, 3-5\% of cases of TAO progress and require more intensive therapy, including glucocorticoid therapy, orbital radiotherapy and orbital decompression surgery (1). As one of the most important non-surgical approaches, glucocorticoid therapy may be administered via the oral, retrobulbar, subconjunctival or intravenous (i.v.) route. Intravenous glucocorticoids have been confirmed to be more effective and much safer than administration via oral and local routes (2). Therefore, the preferred first-line treatment for active moderate-to-severe TAO, as recommended by the European Group on Graves' Ophthalmopathy (EUGOGO), is i.v. glucocorticoid therapy (3). There are two major regimens for i.v. glucocorticoid therapy: one is a weekly protocol and the other is a daily scheme. A recent study has indicated that a weekly protocol was more effective and safer than a daily scheme in a cumulative dose of $4.5 \mathrm{~g}$ i.v. glucocorticoid therapy for moderate-to-severe active TAO (4). However, the details of the treatment schedule are still debatable. More data and further studies are required. On the other hand, the first and most important step prior to starting therapy is to establish whether TAO is severe and whether there is active inflammation (5). To date, no biomarker has been confirmed that is able to definitely assess the activity and severity of TAO and the effect of treatment. Therefore, the primary objective of the present study was to compare the treatment effects between 
the two regimens. The secondary objective was to investigate associated cytokines.

\section{Patients and methods}

Patients. The present study was a single center, prospective, randomized, open-label trial performed at the Third Affiliated Hospital of Sun Yat-sen University (Guangzhou, China) between January 2012 and June 2017. A total of 160 consecutive patients with TAO (including 54 inactive, 16 active mild and 90 active moderate-to-severe), 60 patients with isolated GD without ophthalmopathy and 60 normal controls (NC) were enrolled. The diagnoses of TAO were based on the EUGOGO consensus (3). Patients were excluded if they had infectious diseases, allergies, autoimmune diseases, coronary artery disease, diabetes mellitus, hypertension, liver disease, kidney disease or a history of alcoholism. None of the patients had received any previous steroid treatment, orbital radiotherapy or orbital surgery.

Study design. A total of 90 patients with active moderate-tosevere TAO were randomized at a ratio of $1: 1$ to receive glucocorticoid therapy for 12 weeks on a weekly protocol or daily scheme. Sealed opaque envelopes, which were arranged in a computer-generated random order prepared by a statistician prior to the study, were opened to determine the patients treatment assignments. The weekly protocol was as follows: $0.5 \mathrm{~g}$ methylprednisolone i.v. weekly for 6 weeks and then the dose was tapered by $0.25 \mathrm{~g} /$ week over the following 6 weeks. The daily scheme was as follows: $0.5 \mathrm{~g}$ methylprednisolone i.v. daily for 5 consecutive days, followed by oral methylprednisolone for 3 months. Oral methylprednisolone started at $32 \mathrm{mg} /$ day for 2 weeks and then the dose was tapered by $4 \mathrm{mg} /$ day every 2 weeks. As a result, the cumulative doses of glucocorticoid were $4.5 \mathrm{~g}$ in the weekly protocol and $4.3 \mathrm{~g}$ in the daily scheme $(\mathrm{P}>0.05)$. All patients were required to quit smoking, to take oral anti-thyroid drugs and received local measures for relieving symptoms (including sunglasses, artificial tears, lubricant ointments and prisms) according to advice from specialists. Within the cohort, two patients treated under the weekly protocol and four on the daily scheme were infected with hepatitis B. All of them received antiviral treatments.

Measurement of serum cytokines. Blood samples from all 289 subjects, which consisted of $69 \mathrm{NCs}, 60$ patients with isolated GD and 160 with TAO, were collected at baseline. At the 12-week follow-up visit, blood samples from all 160 subjects with TAO were collected, which comprised 54 patients with inactive TAO, 16 patients with active mild TAO and 90 patients with active moderate-to-severe TAO. Serum samples were centrifuged and stored at $-80^{\circ} \mathrm{C}$ for future analysis. Interleukin (IL)-2, IL-6 and IL-17 concentrations were measured using a solid-phase sandwich ELISA (Neobioscience Technology Co.). The lowest detectable limits of the assays for IL-2, IL-6 and IL-17 were 2.23, 0.7 and $1.10 \mathrm{pg} / \mathrm{ml}$, respectively.

Ophthalmic assessment. Ophthalmic assessments were performed prior to therapy and at the 12-week follow-up visit. The ophthalmic assessments included physical eye examinations (eye symptoms, swelling, conjunctival congestion, diplopia, visual acuity and proptosis) and orbital CT examinations (thickness of the extraocular muscles and orbital fat density). Physical eye examinations were performed by one single ophthalmologist and orbital CT examinations were determined by a single radiologist. Proptosis was measured by a Hertel exophthalmometer. The upper limit of normal for Chinese subjects in the present study was $18.6 \mathrm{~mm}$ (6). Soft tissue involvement was assessed using a color atlas. The thickness of the extraocular muscles was defined as the maximum coronal section area of the most hypertrophic rectus muscle in each eye, as measured by orbital CT. Activity of TAO was assessed via the clinical activity score (CAS) (7). The severity of TAO was assessed according to the EUGOGO (3).

Clinical outcome assessment. The primary clinical outcome was the overall response rate. 'Response' was defined as an improvement in at least two major criteria and one minor criterion. Otherwise, the outcome was considered 'non-response'. The major criteria included the following: i) Improvement in CAS by $\geq 2$ points, ii) improvement in diplopia(disappearance or reduction in degree), iii) improvement in visual acuity by $\geq 1 / 10$ and i.v.) reduction in proptosis or lid width of $\geq 2 \mathrm{~mm}$. The minor criteria were favorable changes in soft tissues and improvement in the patient's self-assessment (8).

The secondary clinical outcomes were the remission rate of major ophthalmic manifestations, average changes in the CAS and adverse events.

Adverse events. To assess the treatment-associated side effects, body weight, blood pressure, routine biochemical analysis and electrocardiogram were measured at baseline and at the 12-week follow-up visit. The measurements were also performed whenever the patient felt uncomfortable. Major adverse events were defined as life-threatening liver failure and cardio- or cerebrovascular complications (5). Minor adverse events included weight gain (an increase of at least $2 \mathrm{~kg}$ at the 12-week follow-up visit compared to baseline), hyperglycemia (fasting blood glucose $\geq 7 \mathrm{mmol} / 1$ or 2 -h postprandial blood glucose $\geq 11.1 \mathrm{mmol} / \mathrm{l}$ ), hypertension (systolic blood pressure $\geq 140 \mathrm{mmHg}$ or diastolic blood pressure $\geq 90 \mathrm{mmHg}$ ), renal dysfunction (estimated glomerular filtration rate $<60 \mathrm{ml} / \mathrm{min} / 1.73 \mathrm{~m}^{2}$ ), hepatic dysfunction (alanine aminotransferase or aspartate aminotransferase elevated by 2.5 -fold above the upper normal limits), gastrointestinal discomfort, sleeplessness and infection.

Statistical analysis. All statistical analyses were performed with SPSS 18.0 (SPSS, Inc.). Continuous variables are expressed as the mean \pm standard deviation or median (interquartile range, 25 th-75th percentile). Categorical variables are expressed as frequencies and percentages. Differences in continuous variables between groups were evaluated by Student's t-test, while differences in categorical variables between groups were analyzed by the $\chi^{2}$ test. Paired Student's t-tests were used to compare continuous variables prior to and after treatment. One-way analysis of variance was applied for comparison of continuous variables among $>2$ groups, which was followed by Fisher's Least Significant Difference post-hoc test in the case of statistical significance. $\mathrm{P}<0.05$ was considered to indicate a statistically significant difference. 


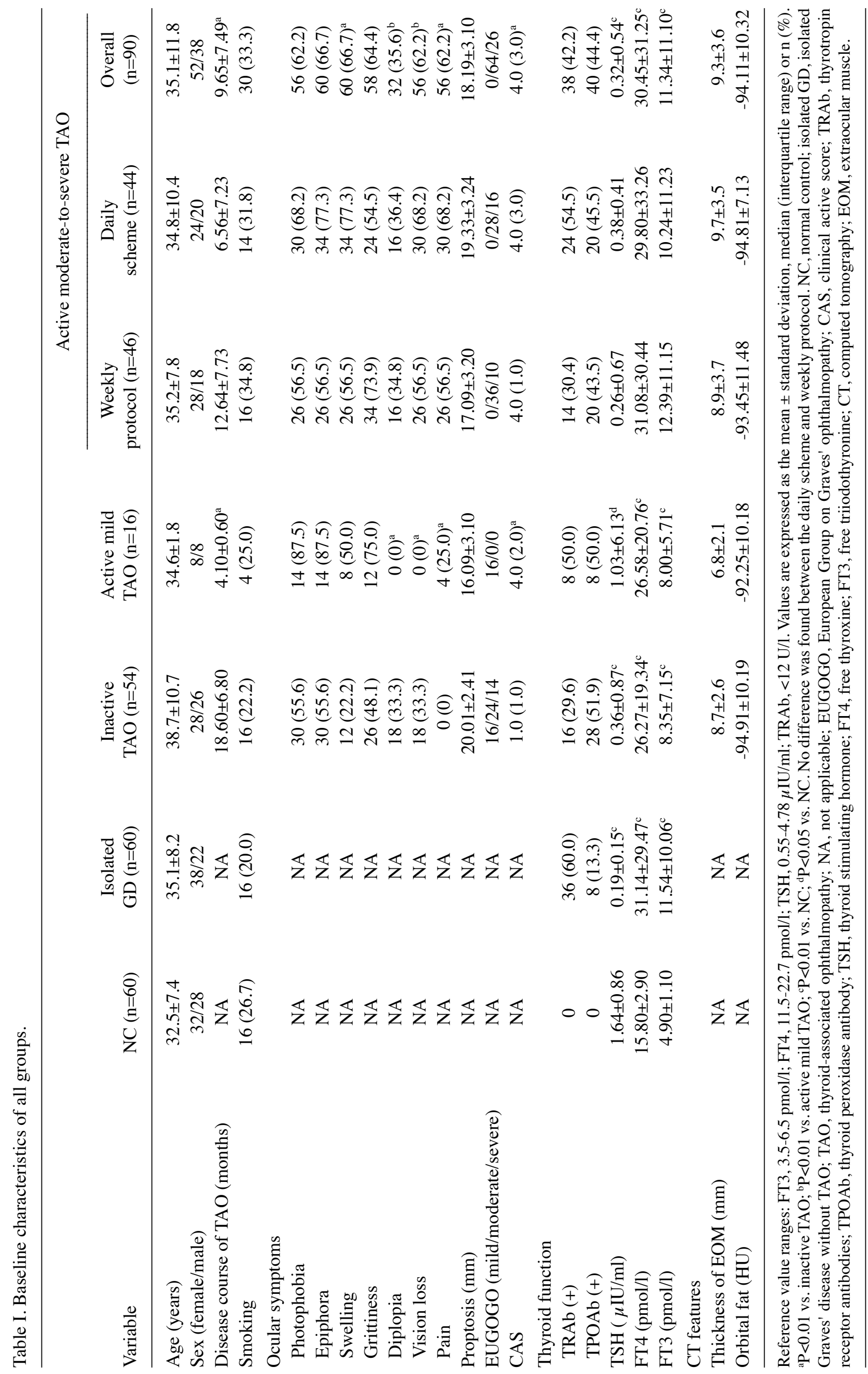


Table II. Comparison of treatment outcomes between the two groups.

\begin{tabular}{|c|c|c|c|c|}
\hline \multirow[b]{2}{*}{ Item } & \multicolumn{2}{|c|}{ Weekly protocol $(n=46)$} & \multicolumn{2}{|c|}{ Daily scheme (n=44) } \\
\hline & Baseline & 12 weeks & Baseline & 12 weeks \\
\hline \multicolumn{5}{|l|}{ Eye symptoms } \\
\hline Photophobia (+/-) & $26 / 20$ & $4 / 42$ & $30 / 14$ & $10 / 34$ \\
\hline Epiphora (+/-) & $26 / 20$ & $8 / 38$ & $34 / 10$ & $10 / 34$ \\
\hline Swelling (+/-) & $26 / 20$ & $8 / 38$ & $34 / 10$ & $6 / 38$ \\
\hline Grittiness (+/-) & $34 / 12$ & $0 / 46$ & $24 / 20$ & $10 / 34$ \\
\hline Diplopia (+/-) & $16 / 30$ & $10 / 36$ ( 2 worsened $)$ & $16 / 28$ & $6 / 38$ \\
\hline Vision loss (+/-) & $26 / 20$ & 10/36 (2 worsened) & $30 / 14$ & $10 / 34$ \\
\hline Pain $(+/-)$ & $26 / 20$ & $12 / 34$ & $30 / 14$ & $6 / 38$ \\
\hline Proptosis (mm) & $17.25 \pm 1.80$ & $17.94 \pm 2.08$ & $20.42 \pm 2.61$ & $20.58 \pm 2.78$ \\
\hline CAS score & $4.0(1.0)$ & $2.0(1.0)^{\mathrm{a}}$ & $4.0(3.0)$ & $2.0(2.0)^{\mathrm{a}}$ \\
\hline \multicolumn{5}{|l|}{ Thyroid function } \\
\hline FT3 (pmol/l) & $12.39 \pm 11.15$ & $5.33 \pm 1.588^{\mathrm{b}}$ & $10.24 \pm 11.23$ & $4.82 \pm 0.87^{\mathrm{b}}$ \\
\hline FT4 (pmol/l) & $31.08 \pm 30.44$ & $15.56 \pm 4.36^{\mathrm{b}}$ & $29.80 \pm 33.26$ & $15.20 \pm 2.94^{\mathrm{b}}$ \\
\hline $\mathrm{TSH}(\mu \mathrm{IU} / \mathrm{ml})$ & $0.26 \pm 0.67$ & $1.72 \pm 1.80^{\mathrm{b}}$ & $0.38 \pm 0.41$ & $2.82 \pm 0.76^{b}$ \\
\hline \multicolumn{5}{|l|}{ CT features } \\
\hline Thickness of EOM (mm) & $8.9 \pm 3.7$ & $8.6 \pm 3.4$ & $9.7 \pm 3.5$ & $9.4 \pm 3.6$ \\
\hline Orbital fat (HU) & $-91.99 \pm 12.49$ & $-95.23 \pm 9.62$ & $-93.92 \pm 7.28$ & $-104.1 \pm 13.15$ \\
\hline
\end{tabular}

Table III. Remission of treatment outcomes between the two groups.

\begin{tabular}{lccc}
\hline Item & Total remission rate $(\mathrm{n}=90)$ & Weekly protocol $(\mathrm{n}=46)$ & Daily scheme $(\mathrm{n}=44)$ \\
\hline Photophobia & $42(75.0)$ & $22 / 26(84.6)$ & $20 / 30(66.7)$ \\
Lacrimation & $42(70.0)$ & $18 / 26(69.2)$ & $24 / 34(70.6)$ \\
Swelling & $46(76.7)$ & $18 / 26(69.2)$ & $28 / 34(82.4)$ \\
Grittiness & $48(82.8)$ & $34 / 34(100.0)$ & $14 / 24(58.3)$ \\
Diplopia & $18(56.2)$ & $8 / 16(50)$ & $10 / 16(62.5)$ \\
Vision loss & $38(67.9)$ & $18 / 26(69.2)$ & $20 / 30(66.7)$ \\
Pain & $38(67.9)$ & $14 / 26(53.8)$ & $24 / 30(80.0)$ \\
\hline
\end{tabular}

Values are expressed as n (\%). No significant difference was found between the daily scheme and weekly protocol.

\section{Results}

Clinical characteristics at baseline. A total of 280 indi-

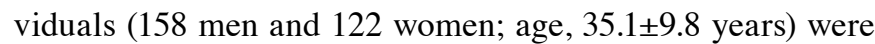
enrolled. A total of $82(29.3 \%)$ individuals were smokers and 198 (70.7\%) were non-smokers. The detailed clinical characteristics at baseline are summarized in Table I. No significant differences were observed in terms of age, sex, or smoking status among all groups. Patients with isolated GD and TAO had similar levels of thyroid hormones $(\mathrm{P}>0.05)$. Free triiodothyronine and free thyroxine levels in these two groups were significantly higher than those in the NC group, whereas thyroid stimulating hormone was significantly lower $(\mathrm{P}<0.01)$.

The duration of eye disease in the subjects with active TAO (either mild or moderate-to-severe) was much shorter than that in the subjects with inactive TAO $(\mathrm{P}<0.01)$. The subjects with active moderate-to-severe TAO were more inclined to experience epiphora and pain in the eyes than the subjects with inactive TAO $(\mathrm{P}<0.01)$. Numerous subjects with active moderate-to-severe TAO suffered from diplopia and vision loss (35.6 and $62.2 \%$, respectively). However, none of the patients with active mild TAO complained of diplopia and decreased vision. Proptosis, thyroid function and ocular CT features were 

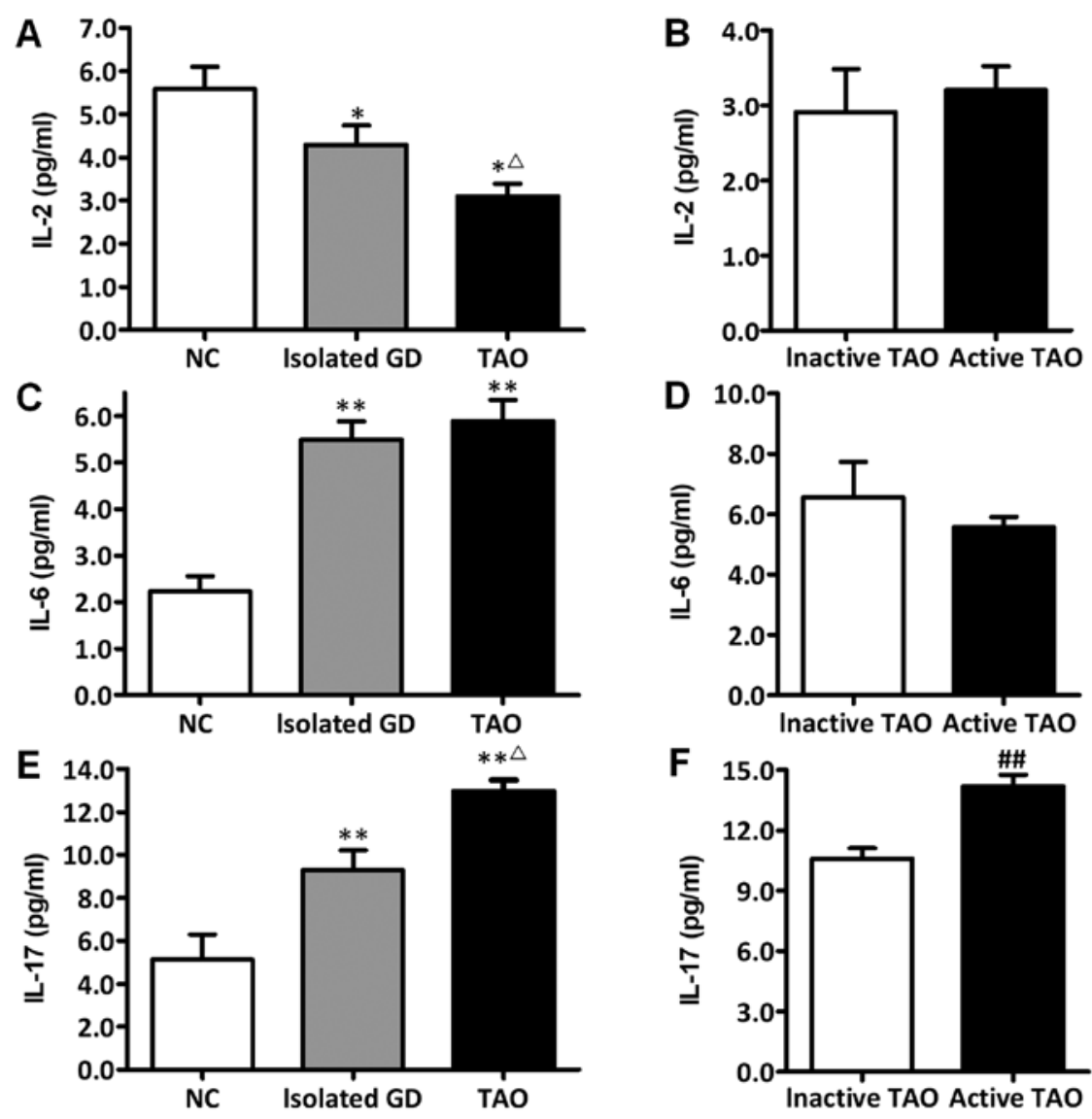

Figure 1. Serumlevels of (A and B) IL-2, (C and D) IL-6 and (E and F) IL-17 at baseline. ${ }^{*} \mathrm{P}<0.05$ vs. NC. ${ }^{* *} \mathrm{P}<0.01$ vs. NC. ${ }^{\Delta} \mathrm{P}<0.05$ vs. isolated GD. ${ }^{\# \#} \mathrm{P}<0.01$ vs. inactive TAO. IL, interleukin; NC, normal control; TAO, thyroid-associated ophthalmopathy; GD, Graves' disease.
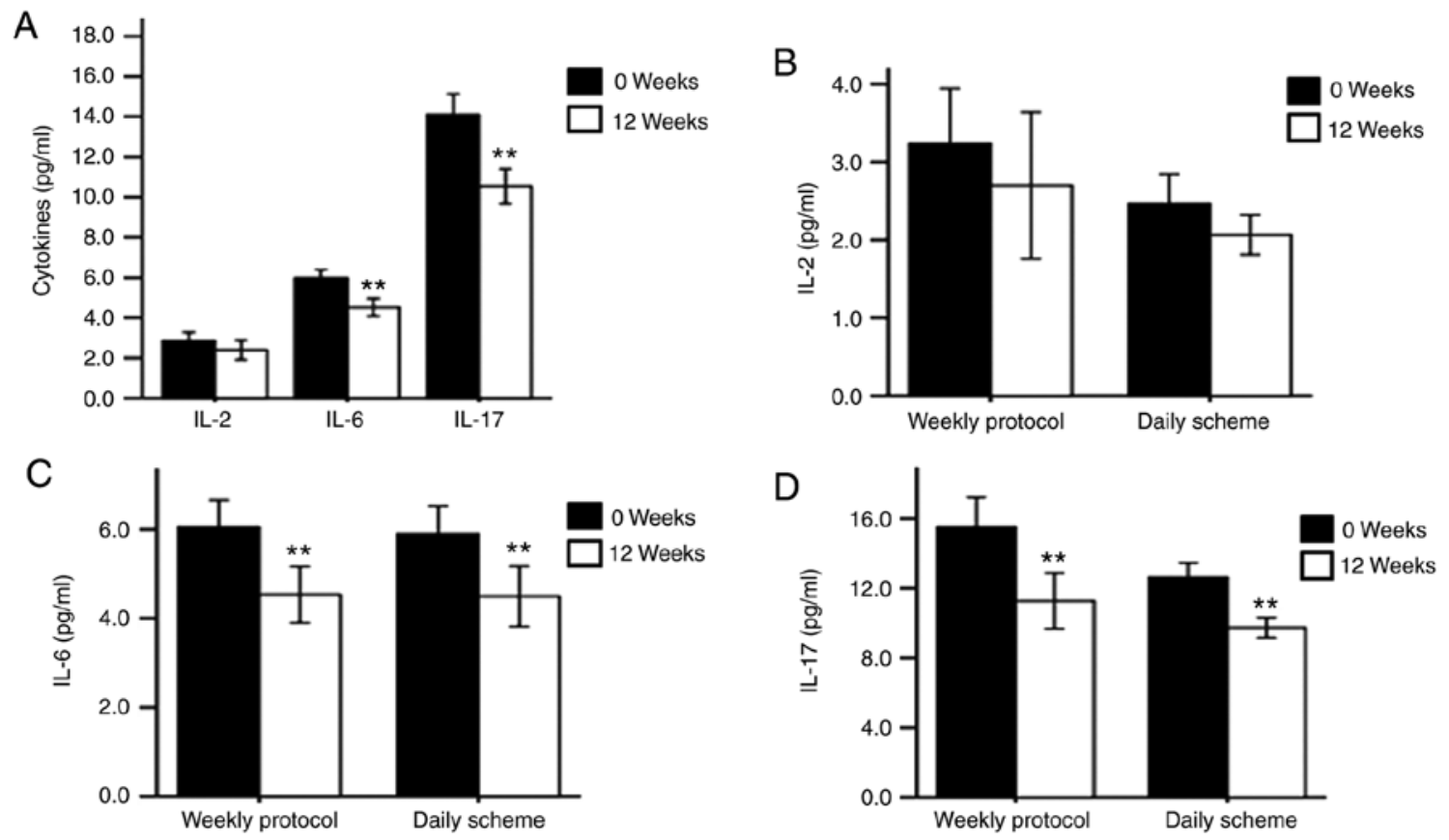

Figure 2. Changes in IL-2, IL-6 and IL-17 at the 12-week follow-up visit compared to baseline. (A) Overall serum IL-2, IL-6 and IL-17 levels in all patients with active moderate-to-severe thyroid-associated ophthalmopathy. Changes in serum (B) IL-2, (C) IL-6 and (D) IL-17 on the weekly protocol and daily scheme. ${ }^{* *} \mathrm{P}<0.01$ vs. 0 weeks. IL, interleukin.

similar among patients with inactive TAO, active mild TAO and active moderate-to-severe TAO $(\mathrm{P}>0.05)$.
There were no significant differences between the patients allocated to the weekly protocol and those allocated to the 
Table IV. Adverse events of glucocorticoid therapy.

\begin{tabular}{lcc}
\hline Item & $\begin{array}{c}\text { Weekly } \\
\text { protocol }\end{array}$ & $\begin{array}{c}\text { Daily } \\
\text { scheme }\end{array}$ \\
\hline Number of adverse events & $16(4.35)$ & $40(11.36)^{\mathrm{a}}$ \\
Patients with adverse events & $12 / 46(26.1)$ & $28 / 44(63.6)^{\mathrm{b}}$ \\
Weight gain & 8 & 4 \\
Hyperglycemia & 0 & 12 \\
Hypertension & 0 & 0 \\
Gastrointestinal discomfort & 4 & 8 \\
Sleeplessness & 4 & 12 \\
Hepatic lesion & 0 & 0 \\
Renal dysfunction & 0 & 0 \\
Infection & 0 & 4 \\
\hline
\end{tabular}

${ }^{\mathrm{a}} \mathrm{P}<0.05$ vs. weekly protocol, ${ }^{\mathrm{b}} \mathrm{P}<0.01$ vs. weekly protocol. Values are expressed as $\mathrm{n}, \mathrm{n}(\%)$ or $\mathrm{n} / \mathrm{total}(\%)$.

daily scheme in terms of clinical variables, biochemical features and ocular CT characteristics $(\mathrm{P}>0.05)$.

Clinical outcomes at the 12-week follow-up visit. The overall response rate was $69.8 \%$ in all patients receiving i.v. glucocorticoid therapy at the 12 -week follow-up visit. The response rate in patients on the daily scheme was higher than that in those on the weekly protocol, although the difference was not significant $(77.8 \%$ vs. $63.6 \%, \mathrm{P}>0.05)$. In addition, patients had similar CAS responses, which decreased significantly from 4 to 2 under the two regimens $(\mathrm{P}<0.01)$, but there was no difference between the two regimens ( $P>0.05$; Table II).

The major ocular symptoms in all patients, which consisted of photophobia (75\%), lacrimation (70\%), swelling (76.7\%), grittiness $(82.8 \%)$, diplopia $(62.5 \%)$, vision loss $(67.9 \%)$ and pain $(67.9 \%)$, improved significantly (Table III). The two regimens had a similar remission rate of major ophthalmic manifestations $(\mathrm{P}>0.05)$. Of note, two patients on the weekly protocol acquired diplopia with vision loss and their conditions worsened. As a result, they received additional i.v. glucocorticoid therapy and they improved gradually with the additional treatment.

Serum cytokine levels at baseline and after glucocorticoid therapy. The serum levels of IL-2, IL-6 and IL-17 at baseline are summarized in Fig. 1. From the NC group to the isolated GO group followed by the TAO group, the IL-2 level decreased gradually $(\mathrm{P}<0.05)$, while the IL-17 level increased progressively $(\mathrm{P}<0.05)$. The IL-6 level in the isolated GD group was similar to that in the TAO group ( $P>0.05)$, both of which were significantly higher than those in the $\mathrm{NC}$ group $(\mathrm{P}<0.05)$.

Among all subjects with TAO, the IL-17 level in the active TAO group (either mild or moderate-to-severe) was higher than that in the inactive TAO group $(\mathrm{P}<0.01)$, whereas neither IL-2 nor IL-6 was different between the active TAO and inactive TAO groups $(\mathrm{P}>0.05)$.

The levels of serum IL-2, IL-6 and IL-17 after glucocorticoid therapy are summarized in Fig. 2. The IL-2 level in the active moderate-to-severe TAO group was not significantly changed at the 12-week follow-up visit $(\mathrm{P}>0.05)$; however, the IL-6 and IL-17 levels were significantly decreased after treatment $(\mathrm{P}<0.05)$. Notably, the IL-6 and IL-17 levels were markedly declined after treatment with either of the two regimens $(\mathrm{P}<0.01)$. Furthermore, the changes in IL-6 and IL-17 were similar between the two regimens $(\mathrm{P}>0.05 ;$ Fig. $2 \mathrm{D})$.

Adverse effects. Intravenous glucocorticoids were fairly well tolerated in the two regimens without any serious adverse events, including serious liver function impairment (liver enzymes $\geq 2.5$ times the upper limit) and cardiovascular events. The total number of patients suffering minor side effects was higher on the daily scheme than that on the weekly protocol $(\mathrm{P}<0.05$; Table IV). Sleeplessness, gastrointestinal discomfort and hyperglycemia were the most common adverse events on the daily scheme, while weight gain was relatively common on the weekly protocol. Blood pressure and liver and renal function parameters remained normal under the two regimens.

\section{Discussion}

The present study was a single center prospective randomized trial in which the efficacy and safety of two regimens of i.v. glucocorticoid were compared. In this trial, the improvement in clinical manifestations, including photophobia, epiphora, swelling, grittiness, diplopia, vision loss, pain, proptosis and CAS score, and thyroid function and CT features on extraocular muscle after 12-week treatment were the primary outcomes and the levels of three cytokines were also investigated at the same time. The results suggested that the daily scheme had a higher response rate than the weekly protocol, although the difference was not significant and that these two regimens yielded similar improvements in serum cytokines. Numerous minor side effects were recorded in patients on the daily scheme, whereas two patients worsened on the weekly protocol.

Although the pathogenesis of TAO is remains to be fully elucidated, glucocorticoid therapy is the recommended first-line treatment for TAO (9). Glucocorticoid therapy may be administered via oral, local (retrobulbar or subconjunctival) and i.v. routes. Studies have indicated that high-dose glucocorticoids administered via the i.v. route are more effective and better tolerated than those taken orally (10-12). There are various schedules for i.v. glucocorticoid therapy. A recent study indicated that a weekly protocol $(4.5 \mathrm{~g}$ of i.v. glucocorticoid therapy) is safer and more effective than a daily scheme (4). However, the present results suggested that the daily scheme and the weekly protocol were effective for active TAO and that the daily scheme had a relative higher response rate than that of the weekly protocol, although the difference was not statistically significant. Why did the response rate in the present study differ that in the previous study? This may be due to the different details of the daily scheme. In a previous study, i.v. glucocorticoid was administered for 3 consecutive days per week for 4 weeks (4). However, in the present trial, i.v. glucocorticoid was administered for 5 consecutive days. It is possible that extending the treatment time of i.v. glucocorticoid intensified immunosuppression, which leads to favorable effects (5). The difference in the selection of patients may be another reason (5). The response rate was $77.8 \%$ on 
the daily scheme and $63.6 \%$ under the weekly protocol in the present study. Although the difference appeared obvious, it was not statistically significant. Future studies with larger sample size and longer study duration would be required to determine whether there was truly no difference or whether the 12-week follow-up period was not long enough or whether a total of 90 patients was not large enough to reach statistical significance in the present study.

The two regimens were able to improve soft tissue inflammation. However, neither regimen improved extraocular muscle dysfunction and proptosis. This may be due to two reasons. One was the short follow-up time. Improvement in extraocular muscle dysfunction and proptosis requires more time than amelioration of soft tissue inflammation. Another reason was probably the fibrosis in the extraocular muscles, which is more difficult to resolve than edema. Although certain studies indicated that glucocorticoid therapy improved extraocular muscle dysfunction and proptosis $(5,13,14)$, other studies were in agreement with the present results $(10-12,15,16)$. A systematic review also indicated that glucocorticoid therapy had a limited effect on proptosis (5). Further studies are required to clarify these effects.

Generally, intravenous glucocorticoids are fairly well tolerated. Cardiovascular risk and hepatotoxicity are the most severe adverse effects of high-dose glucocorticoids (3-5). In the present study, no cardiovascular events or impaired liver function was recorded in the subjects on either the weekly protocol or daily scheme. The daily scheme had a slightly higher incidence of total adverse effects than that of the weekly protocol. However, the deterioration of eye symptoms was only recorded on the weekly protocol. Thus, the present results indicated that the two regimens had their own merits.

TAO is regarded as an autoimmune disease (1). Glucocorticoid therapy is a well-established treatment for TAO owing to its anti-inflammatory and immunosuppressive actions (9). Previous studies have focused on T lymphocytes, particularly $\mathrm{CD} 4^{+} \mathrm{T}-$ helper $(\mathrm{Th})$ cells, which are the principal immune effector $\mathrm{T}$ cells $(17,18)$. Activated $\mathrm{CD} 4{ }^{+} \mathrm{Th}$ cells differentiate into Th1, Th2, Th17 and Treg subsets, which produce numerous cytokines (17). These cytokines have been proven to have an important role in TAO $(3,19,20)$. Accordingly, cytokines that regulate inflammation have the potential to be biomarkers to evaluate the activity of TAO and the efficacy of treatment options. IL-2 is a characteristic cytokine of Th1 T cells and IL-6 is one of the key cytokines secreted by Th2 T cells (19). In addition, IL-17 is the signature cytokine of Th17 T cell-derived population with highly pro-inflammatory properties (21). It is considered to have a significant role in numerous autoimmune diseases, including rheumatoid arthritis and systemic lupus erythematosus $(22,23)$. Therefore, IL-2, IL-6 and IL-17 were selected in the present study.

In the present study, the IL-2 levels in the TAO and isolated GD groups were lower than those in the NC group. The IL-6 and IL-17 levels in the TAO and isolated GD groups were higher than those in the NC group. These results implied that all three cytokines are involved in GD. Previous studies suggested that cytokines produced by Th1 T cells were dominant at the early stage and in active TAO, whereas those secreted by $\mathrm{Th} 2 \mathrm{~T}$ cells had a greater role in later stages and in inactive TAO $(24,25)$. Certain previous studies reported that IL-2 and IL-6 levels in active TAO were higher than those in inactive TAO (26-29). However, this result was not supported by other reports $(4,30,31)$. In the present study, IL-2 and IL-6 levels in active TAO were similar to those in inactive TAO. Taken together, the present results indicated that neither IL-2 nor IL-6 was an ideal biomarker for the evaluation of TAO. Notably, the IL-17 level was significantly higher in the TAO group than that in the isolated GD and NC groups. In addition, the IL-17 concentration in the active TAO group was higher than that in the inactive TAO group, which is consistent with previous reports (32-34). Furthermore, the IL-17 level was markedly decreased at the 12-week follow-up visit after glucocorticoid therapy under the two regimens. The present results are in line with those of a previous study (32). Evidence has indicated that IL-17 has a key role in the pathogenesis of autoimmune thyroiditis and GD (35-38). These data suggested that IL-17 may reflect the inflammatory state and be a potential indicator for the severity and activity of TAO. However, a recent study indicated that the IL-17 concentration was elevated in GD with/without TAO but reduced in active and inactive TAO (31), which was not in agreement with previous studies. Given that IL-17 has only recently been studied, more data should be accumulated.

The present study assessedIL-2, IL-6 and IL-17 to provide novel insight into TAO. Considering the complexity of the immunopathogenesis and diversity of cytokines, further studies are required. The sample size should be enlarged in future studies. Furthermore, other cytokines produced by infiltrating immunocompetent cells and orbital fibroblasts should be investigated to help understand the pathogenesis of TAO more comprehensively.

In conclusion, the present results provide evidence indicating that: i) For patients with active moderate-to-severe TAO, the daily scheme of intravenous glucocorticoids had a higher response rate than the weekly protocol and these two regimens had their own merits with regard to adverse effects; and ii) IL-17 has the potential to serve as a marker for evaluating the activity of TAO and the efficacy of treatment options.

\section{Acknowledgements}

The authors thank the statistician Dr Lian-Xiong Yuan from the Third Affiliated Hospital of Sun Yat-sen university for his help with the statistical analysis.

\section{Funding}

The current study was funded by National Key R\&D Program of China (grant no. 2017YFA0105803), the General Program of National Natural Science Foundation of China (grant no. 81770826), the 5010 Clinical Research Projects of Sun Yat-sen University (grant no. 2015015), the Science and Technology Plan Projects of Guangdong Province (grant no. 2016A050502010), the Key Special Projects of Medical and Health Collaborative Innovation of Guangzhou City (grant no. 201604020016) and the Special Scientific Research Project of Guangzhou City (grant no. 2060404). The funding bodies had no role in the design of the study, the collection, analysis and interpretation of data or the writing of the manuscript. 


\section{Availability of data and materials}

The datasets used and/or analyzed during the current study are available from the corresponding author on reasonable request.

\section{Authors' contributions}

YC conceived the study, designed the research plan, supervised and supported the study, verified data and statistical analysis results, provided guidance with the writing, had full access to all data in the study and took responsibility for the integrity of the data and the accuracy of the data analysis. PM and XT performed the study, collected and analyzed data, wrote and revised the manuscript and contributed to the interpretation of results. YW, SL, MW, QY, JS, BZ, JL, LiZ and LoZ followed up with the participants, collected data and participated in data analysis and interpretation. Additionally, they revised the manuscript. All authors read and approved the final manuscript.

\section{Ethics approval and consent to participate}

The current study was approved by the Ethics Committee of the Third Affiliated Hospital of Sun Yat-sen University, Guangzhou, P.R. China and written informed consent was obtained from all subjects.

\section{Patient consent for publication}

Not applicable.

\section{Competing interests}

The authors declare that they have no competing interests.

\section{References}

1. Bartalena L, Pinchera A and Marcocci C: Management of Graves' ophthalmopathy: Reality and perspectives. Endocr Rev 21: 168-199, 2000.

2. Gao G, Dai J, Qian Y and Ma F: Meta-analysis of methylprednisolone pulse therapy for Graves' ophthalmopathy. Clin Exp Ophthalmol 42: 769-777, 2014.

3. Bartalena L, Baldeschi L, Dickinson AJ, Eckstein A, Kendall-Taylor P, Marcocci C, Mourits MP, Perros P, Boboridis K, Boschi A, et al: Consensus statement of the European group on Graves' orbitopathy (EUGOGO) on management of Graves' orbitopathy. Thyroid 18: 333-346, 2008.

4. Zhu W, Ye L, Shen L, Jiao Q, Huang F, Han R, Zhang X, Wang S, Wang $\mathrm{W}$ and Ning G: A prospective, randomized trial of intravenous glucocorticoids therapy with different protocols for patients with Graves' ophthalmopathy. J Clin Endocrinol Metab 99: 1999-2007, 2014.

5. Zang S, Ponto KA and Kahaly GJ: Clinical review: Intravenous glucocorticoids for Graves' orbitopathy: Efficacy and morbidity. J Clin Endocrinol Metab 96: 320-332, 2011.

6. Tsai CC, Kau HC, Kao SC and Hsu WM: Exophthalmos of patients with Graves' disease in Chinese of Taiwan. Eye (Lond) 20: 569-573, 2006.

7. Mourits MP, Koornneef L, Wiersinga WM, Prummel MF, Berghout A and van der Gaag R: Clinical criteria for the assessment of disease activity in Graves' ophthalmopathy: A novel approach. Br J Ophthalmol 73: 639-644, 1989.

8. Bartalena L, Marcocci C, Bogazzi F, Manetti L, Tanda ML, Dell'Unto E, Bruno-Bossio G, Nardi M, Bartolomei MP, Lepri A, et al: Relation between therapy for hyperthyroidism and the course of Graves' ophthalmopathy. N Engl J Med 338: 73-78, 1989.
9. Modjtahedi SP, Modjtahedi BS, Mansury AM, Selva D, Douglas RS, Goldberg RA and Leibovitch I: Pharmacological treatments for thyroid eye disease. Drugs 66: 1685-1700, 2006.

10. Marcocci C, Bartalena L, Tanda ML, Manetti L, Dell'Unto E, Rocchi R, Barbesino G, Mazzi B, Bartolomei MP, Lepri P, et al: Comparison of the effectiveness and tolerability of intravenous or oral glucocorticoids associated with orbital radiotherapy in the management of severe Graves' ophthalmopathy: Results of a prospective, single-blind, randomized study. J Clin Endocrinol Metab 86: 3562-3567, 2001.

11. Macchia PE, Bagattini M, Lupoli G, Vitale M, Vitale G and Fenzi G: High-dose intravenous corticosteroid therapy for Graves' ophthalmopathy. J Endocrinol Invest 24: 152-158, 2001.

12. Kauppinen-Makelin R, Karma A, Leinonen E, Loyttyniemi E, Salonen O, Sane T, Setala K, Viikari J, Heufelder A and Valimaki M: High dose intravenous methylprednisolone pulse therapy versus oral prednisone for thyroid-associated ophthalmopathy. Acta Ophthalmol Scand 80: 316-321, 2002.

13. Kahaly GJ, Pitz S, Hommel G and Dittmar M: Randomized, single blind trial of intravenous versus oral steroid monotherapy in Graves' orbitopathy. J Clin Endocrinol Metab 90: 5234-5240, 2005.

14. van Geest RJ, Sasim IV, Koppeschaar HP, Kalmann R, Stravers SN, Bijlsma WR and Mourits MP: Methylprednisolone pulse therapy for patients with moderately severe Graves' orbitopathy: A prospective, randomized, placebo-controlled study. Eur J Endocrinol 158: 229-237, 2008.

15. Ng CM, Yuen HK, Choi KL, Chan MK, Yuen KT, Ng YW and Tiu SC: Combined orbital irradiation and systemic steroids compared with systemic steroids alone in the management of moderate-to-severe Graves' ophthalmopathy: A preliminary study. Hong Kong Med J 11: 322-330, 2005.

16. Aktaran S, Akarsu E, Erbağci I, Araz M, Okumus S and Kartal M: Comparison of intravenous methylprednisolone therapy vs. oral methylprednisolone therapy in patients with Graves' ophthalmopathy. Int J Clin Pract 61: 45-51, 2007.

17. Huang Y, Fang S, Li D, Zhou H, Li B and Fan X: The involvement of T cell pathogenesis in thyroid-associated ophthalmopathy. Eye (Lond) 33: 176-182, 2019.

18. Khong JJ, McNab AA, Ebeling PR, Craig JE and Selva D: Pathogenesis of thyroid eye disease: Review and update on molecular mechanisms. Br J Ophthalmol 100: 142-150, 2016.

19. Gianoukakis AG, Khadavi N and Smith TJ: Cytokines, Graves' disease, and thyroid-associated ophthalmopathy. Thyroid 18: 953-958, 2008.

20. Douglas RS and Gupta S: The pathophysiology of thyroid eye disease: Implications for immunotherapy. Curr Opin Ophthalmol 22: 385-390, 2011.

21. Mills KH: Induction, function and regulation of IL-17-producing T cells. Eur J Immunol 38: 2636-2649, 2008.

22. Ziolkowska M, Koc A, Luszczykiewicz G, KsiezopolskaPietrzak K, Klimczak E, Chwalinska-Sadowska $\mathrm{H}$ and Maslinski W: High levels of IL-17 in rheumatoid arthritis patients: IL-15 triggers in vitro IL-17 production via cyclosporin A-sensitive mechanism. J Immunol 164: 2832-2838, 2000.

23. Nalbandian A, Crispin JC and Tsokos GC: Interleukin-17 and systemic lupus erythematosus: Current concepts. Clin Exp Immunol 157: 209-215, 2009.

24. Yang D, Hiromatsu Y, Hoshino T, Inoue Y, Itoh K and Nonaka K: Dominant infiltration of $\mathrm{T}(\mathrm{H}) 1$-type $\mathrm{CD} 4+\mathrm{T}$ cells at the retrobulbar space of patients with thyroid-associated ophthalmopathy. Thyroid 9: 305-310, 1999.

25. Aniszewski JP, Valyasevi RW and Bahn RS: Relationship between disease duration and predominant orbital $\mathrm{T}$ cell subset in Graves' ophthalmopathy. J Clin Endocrinol Metab 85: 776-780, 2000.

26. Wakelkamp IM, Bakker O, Baldeschi L, Wiersinga WM and Prummel MF: TSH-R expression and cytokine profile in orbital tissue of active vs. inactive Graves' ophthalmopathy patients. Clin Endocrinol (Oxf) 58: 280-287, 2003.

27. Hiromatsu Y, Kaku H, Miyake I, Murayama S and Soejima E: Role of cytokines in the pathogenesis of thyroid-associated ophthalmopathy. Thyroid 12: 217-221, 2002.

28. Avunduk AM, Avunduk MC, Pazarli H, Oguz V, Varnell ED, Kaufman HE and Aksoy F: Immunohistochemical analysis of orbital connective tissue specimens of patients with active Graves ophthalmopathy. Curr Eye Res 30: 631-638, 2005.

29. Lacka K, Manuszewska E, Korczowska I and Lacki JK: The effect of methylprednisolone pulse treatment on cytokine network in Graves ophthalmopathy. Curr Eye Res 32: 291-297, 2007. 
30. Esfahanian F, Naimi E, Doroodgar F and Jadali Z: Th1/Th2 cytokines in patients with Graves' disease with or without ophthalmopathy. Iran J Allergy Asthma Immunol 12: 168-175, 2013.

31. Shen J, Li Z, Li W, Ge Y, Xie M, Lv M, Fan Y, Chen Z, Zhao D and Han Y: Th1, th2, and th17 cytokine involvement in thyroid associated ophthalmopathy. Dis Markers 2015: 609593, 2015.

32. Kim SE, Yoon JS, Kim KH and Lee SY: Increased serum interleukin-17 in Graves' ophthalmopathy. Graefes Arch Clin Exp Ophthalmol 250: 1521-1526, 2012.

33. Peng D, Xu B, Wang Y, Guo $\mathrm{H}$ and Jiang Y: A high frequency of circulating th 22 and th17 cells in patients with new onset Graves' disease. PLoS One 8: e68446, 2013.

34. Li JR, Hong FY, Zeng JY and Huang GL: Functional interleukin-17 receptor A are present in the thyroid gland in intractable Graves disease. Cell Immunol 281: 85-90, 2013.
35. Figueroa-Vega N, Alfonso-Perez M, Benedicto I, Sanchez-Madrid F, Gonzalez-Amaro R and Marazuela M: Increased circulating pro-inflammatory cytokines and Th17 lymphocytes in Hashimoto's thyroiditis. J Clin Endocrinol Metab 95: 953-962, 2010.

36. Horie I, Abiru N, Saitoh O, Ichikawa T, Iwakura Y, Eguchi K and Nagayama Y: Distinct role of T helper Type 17 immune response for Graves' hyperthyroidism in mice with different genetic backgrounds. Autoimmunity 44: 159-165, 2011.

37. Inoue $\mathrm{N}$, Watanabe $\mathrm{M}$, Nanba T, Wada $\mathrm{M}$, Akamizu $\mathrm{T}$ and Iwatani Y: Involvement of functional polymorphisms in the TNFA gene in the pathogenesis of autoimmune thyroid diseases and production of anti-thyrotropin receptor antibody. Clin Exp Immunol 156: 199-204, 2009.

38. Bedoya SK, Lam B, Lau K and Larkin J III: Th17 cells in immunity and autoimmunity. Clin Dev Immunol 2013: 986789, 2013. 